\title{
Study on the Armored and Mechanized Force Command Information System Model Based on the Complex Network
}

\author{
Yucai Dong \\ Institute of Nonlinear Science, Academy of Armored Force Engineering, Beijing 100072, China \\ E-mail: dongyucai@sina.com \\ Guoliang He \\ Department of the Scientific Research, Academy of Armored Force Engineering, Beijing 100072, China \\ Lianghai Yi, Gehua Fan \& Renmou Cai \\ Institute of Nonlinear Science, Academy of Armored Force Engineering, Beijing 100072, China
}

\begin{abstract}
Received: November 29, 2010 Accepted: February 24, 2011 doi:10.5539/jmr.v3n2p230
The research is supported by the Strategic Investment Project of the PlaceTypeplaceAcademy of PlaceNameArmoured Force Engineering (No. 2009ZL05). (Sponsoring information)
\end{abstract}

\begin{abstract}
The wide application of the information technology and the computer network in the military domain has hastened the born of the informationalized operation, and completely changed many characteristics of the limited communication of the mechanized operation, the big-scale operation, and the centralized command control and decision. Facing increasing complexity and network structure characteristics of the command information system, the research idea based on the complex network is proposed in this article, and the topology model of the command information system is established by mapping the three-dimensional structure to the two-dimensional link. By the method of the entity numerical analysis, the existence of the small world and the scale-free characteristics is tested and verified, which could provide positive theoretical reference for the construction of the future system of the armored and mechanized force.
\end{abstract}

Keywords: Complex network, Armored and mechanized force, Command information system, Topology model, Small world, Scale-free

The traditional operation mode of the armored and mechanized force still is that the single military service unit to implement the operation plan and action, and because of the support of the network information technology, the flow of information will not pass the command institution, and the direct cooperation among same-level command institutions will be more easily achieved, and the approach of information flow will increase, and the flow route becomes shorter, and the network effect has been largely enhanced, and the total force of the operation forces will be exerted more easily, and better operation effect could be achieved (Zhu, 2001, P.9-13). With the development of the information technology, the modern informationalized operation mode needs to be further studied. The complex network has been the front and hotspot researched by the present science cycle (Miao, 2001m, P.3-9 \& Li, 2006, P.111-119 \& Jin, 2000, P.1-5), and the research about the complex network is one part of the theoretical research of the complexity, and as the powerful tool to study the complex science and the complex system, the complex network provides new view for the research of the complexity, and its theories have been widely applied in various domains of the society. The complex network theory is used in this article to study the command information system model of the armored and mechanized force, and explore the command characteristics and rules of the armored and mechanized force in the informationalized condition.

\section{The topology model of the command information system}

\subsection{Traditional war network topology model}

According to the practical data, the traditional operation network basically follows the node link principle of "corps - division (brigade) - regiment - battalion - company", and the operation network presents the tree structure, and the information transfer among different operation units is very simple, i.e. the superincumbent transfer mode. The tree network is a kind of regulated network, which means that there is a unique route between any two nodes in the network. The layer of the root node (the highest node) in the tree network is 0 , and the layer of the node under the root node is 1 , and so forth. But if troops are all in the communication range of the communication equipments, they can transfer information each other. In the traditional operation formation, the information communication must pass the command institution, and the information communication among same-level command institutions must pass the upper level command institution, which would induce single information flow route, long information flow path, low network loop efficiency from finding 
the target to attacking the target, and lower total efficiency of the operation force.

\subsection{Command information system network topology model}

To apply the complex network to study the informationalized war, the topology structure of the complex network in the informationalized war should be simulated first by the computer method. It is very important to combine some representative network models into a complex network model for the complex network topology model in the networking war. In the informationalized war, the complexity of the war is unprecedented. The network topology structure of the informationalized war could not completely give up the network topology structure of the traditional operation, but comparing with that, it is more beneficial to information transfer, with shorter average route and many decision information centers. With the demand of the war, the network topology structure of the informationalized war would embody different types of network characteristics. Based on the development rule of the war network, the ideas establishing the model are proposed in this article based on the traditional war topology model.

Rule 1: The entity units are abstracted as nodes and the relationship among units are abstracted as leg, without considering the individual difference and the leg weights.

Rule 2: On each layer of the model, the random network is established.

Rule 3: Only if the information-flow passes any two nodes, the undirected leg link would be operated, and the leg weight value is noted as 1 .

According to above ideas, the tool software Pajeck is used to establish the following information command system topology model.

To study the property of the network topology model, this network is denoted as a matrix. Supposed that $N$ is the total number of all nodes, and the model can be described by the matrix $M$ of $N \times N$, and the value of the matrix element $M_{i j}$ is defined as the state whether the node $i$ is connected with the node $j$, and if it is connected, the value is 1 , or else, it is 0 , and if the self-loop is not considered, the values on the diagonals of the matrix are all 0 , and in addition, the degree $K$ of the node $i$ is defined as the number of the nodes that the network distance to the node $i$ is 1 .

\section{Statistical analysis of the complex network characteristics}

\subsection{Analysis of scale-free characteristic}

In the entity network topology chart, the similarity of two models is that the link amount of most nodes is less, but the link amount of less nodes is more, which is not only consistent with the basic characteristic of the complex network, i.e. scalefree, but also accords with the design standard of the command information system, for example, the senior command control unit has more responsibility advantages of command and perception, so the link has certain characteristic of centralization, but the ending radar perception unit only reports to the senior unit or shares the information in the small range, so the node degree is less. The degree distribution reflects the link of nodes in the network topology, and it is one of important characteristics to distinguish the random network and the rule network. The statistical result of the degree distribution of the experiment entity is seen in Figure 3.

From Figure 3, the degree distribute of the entity 1 presents certain nonhomogeneity, and the power law distribution is not obvious, and the power law distribution of the entity 2 is not clear too, but the basic tendency still exists, the scale factor value is 1.02 by fitting the degree distribution curve through the nonlinear least square method, but at the same time, the coupling effect is not ideal, which is not only because of the statistical scale of the experiment data, but because that the scale-free degree of the system is not high.

\subsection{Analysis of scale-free characteristic}

The statistics of the corresponding average route lengths and cohesion coefficients of the entity topology model is seen in Table 1.

From the Table 1, the average rout length of the entity model 2 is 2.76282 . Comparing with the average route length of the traditional war network topology model, the average route length of this model is less obviously, which would directly reduce the approaches of information processing and transferring, and the information transfer among nodes would be faster, and the information sharing of the whole network would be easier. At the same time, the cohesion efficient of the entity 1 is small, but the cohesion efficient of the entity 2 is larger than the entity 1 , which is mainly because that the cooperative operation and the information sharing have been emphasized in recent years. Comparing with the regulated network and the common random network, the cohesion coefficient of this model is big, which means that the interior association of operation network in the networking war is close and frequent, and the sharing speed of information resources could be enhanced effectively. Big cohesion coefficient and shorter average route mean that this model has the characteristic of the small world network, which could enhance the resource sharing degree. By comparing with the corresponding parameters of various network topologies from literatures, it can be seen that the entity 1 is similar with the traditional tree topology structure, and the entity 2 is basically similar with the characteristic of the small world 


\section{phenomenon.}

\section{Conclusions}

Based on the analysis of traditional tree operation network, a new method is proposed in this article to establish the informationalized war network topology structure model. Starting from the research view of the complex network, the command information system topology model of the armored and mechanized force is established by transforming the entity relationship to the network link. By taking the tank simulation battalion as the research object, the statistics of many parameters such as the average route length, the cohesion coefficient, and the degree distribution reflected by the system could be computed based on the computer simulation, and the characteristics of small world and scale free contained in the system are validated. The result basically accords with the practical development by comparing with the actuality and the target of the informationalized construction of the force, and will provide actively theoretical references for applying the research result about complex network in the future system construction of the armored and mechanized force.

\section{References}

Jin, Wulun \& Guo, Yuanlin. (2000). The Sciences of Complexity and Their Evolutions. Complex Systems and Complexity Science. No.1 (1). P.1-5.

Li, Deyi, Wang, Xinzheng \& Hu, Gangfeng. (2006). Network War and Complex Network. China Military Science. No.19 (4). P.111-119.

Miao, Dongsheng. (2001). Current Situation and Prospect of Complexity Research. Journal of Systemic Dialectics. No.9 (4). P.3-9.

Zhu, Nanzhi \& Zhu, Decheng. (2001). Command Automation System Engineering. Beijing: Electronic Industry Press. P.9-13.

Table 1. Corresponding average route lengths and cohesion coefficients of the entity topology model

\begin{tabular}{|l|l|l|}
\hline Research object & Average route length & Cohesion coefficient \\
\hline Entity 1 & 3.80513 & 0.06325 \\
\hline Entity 2 & 2.76282 & 0.14425 \\
\hline
\end{tabular}

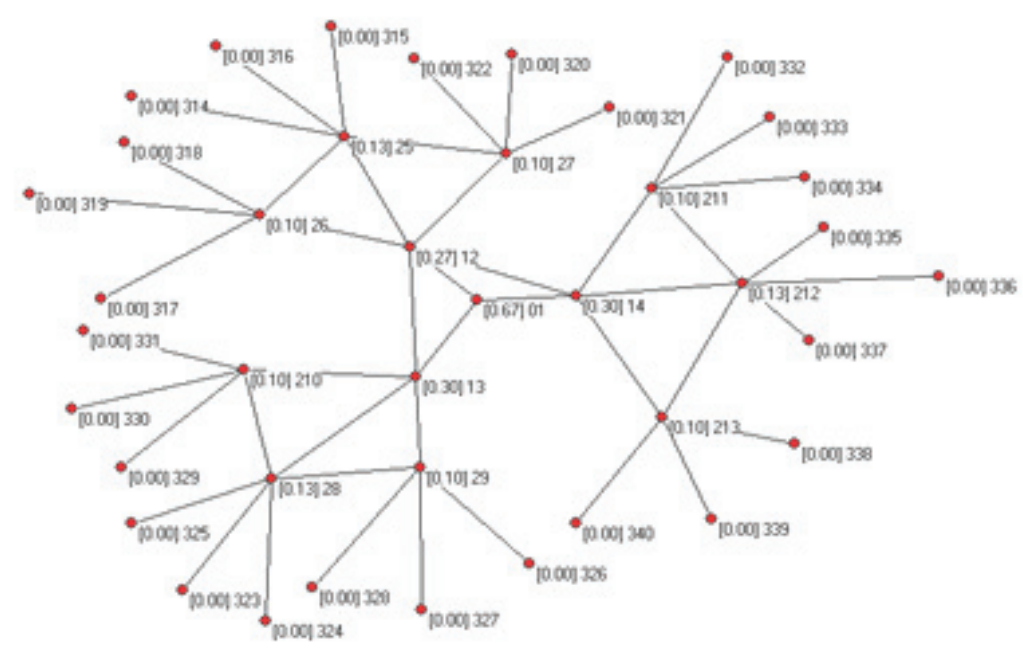

Figure 1. Traditional War Network Topology Model 


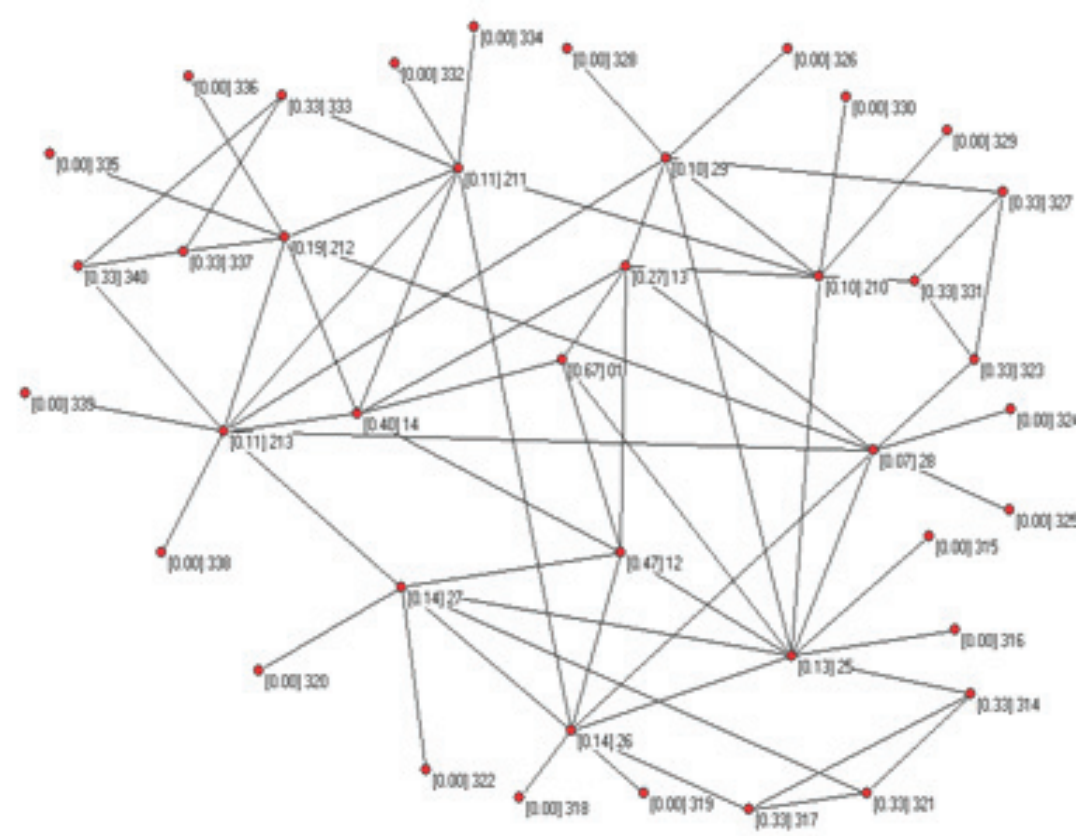

Figure 2. Network Topology Model of the Command Information System

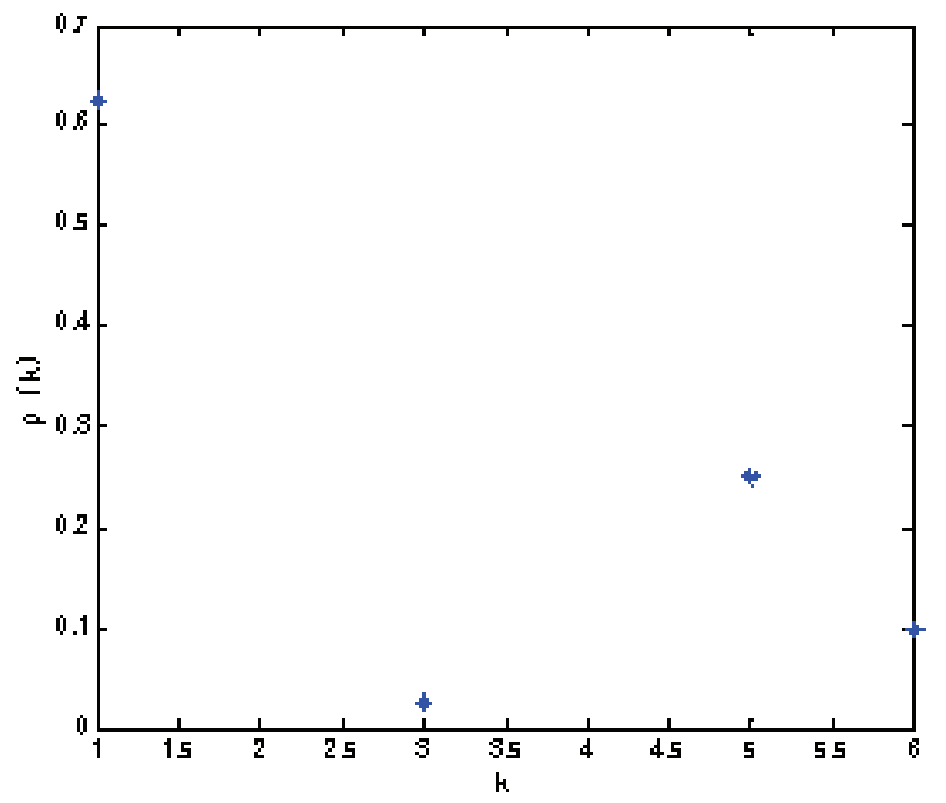

Figure 3. Degree Distribution Statistics of the Traditional Model 


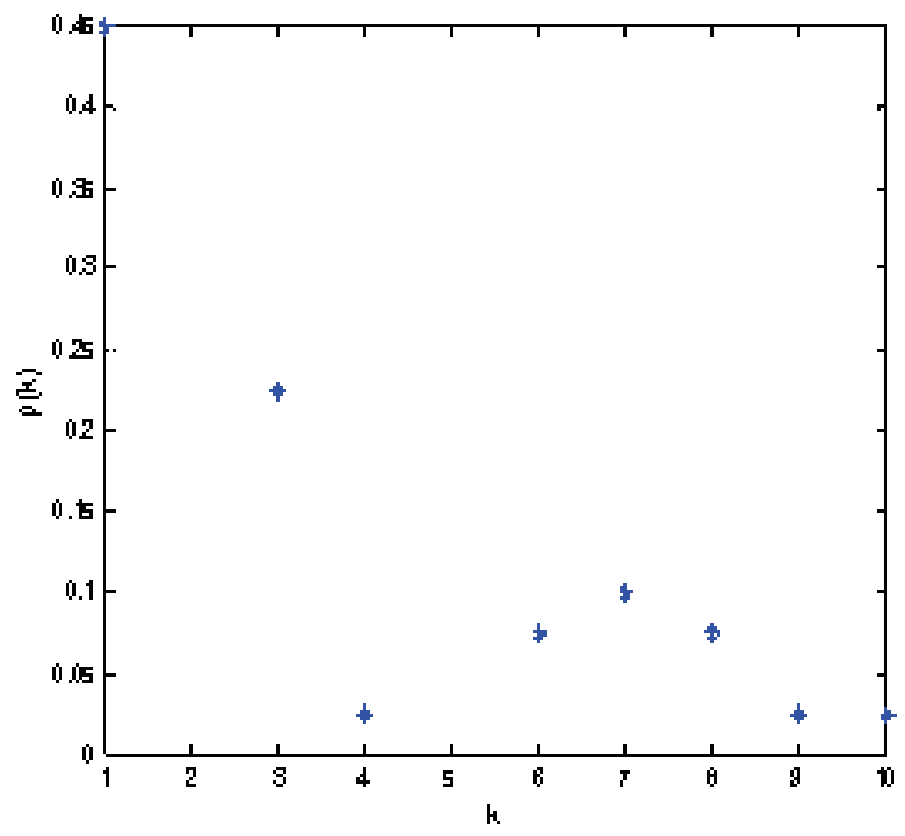

Figure 4. Degree Distribution Statistics of the Network Model 